\title{
Investigation of the Hydrodynamic Behaviour of Particles and Aggregates by Particle Image Velocimetry (PIV)
}

\author{
F. Xiao, X. Y. Li* and K. M. Lam
}

Department of Civil Engineering, The University of Hong Kong, Pokfulam Rd., Hong Kong, China (E-mail:feng_XIAO@hkusua.hku.hk;xlia@hkucc.hku.hk;kmlam@hkucc.hku.hk)

\begin{abstract}
An advanced fluid visualisation technique, particle image velocimetry, was employed to investigate the hydrodynamic properties of particles and aggregates. The experiments were conducted in a settling column filled with a suspension of fluorescent polymeric beads as flow tracers. A thin vertical section of the settling column was illuminated by a laser sheet, while the motions of particles were recorded by a high speed CCD camera. Large solid spheres, flocs of latex microspheres and aggregates of marine diatoms were characterised for their hydrodynamic properties. The trajectories of the tracer particles were tracked when a large particle or aggregate settled through the suspension of the tracers, which gave the streamlines surrounding the falling object. The streamlines demonstrate directly the curvilinear feature of the interaction between approaching particles. However, the available curvilinear model underestimates the collision frequency function of particles by one order of magnitude or two. The collision potentials of the flocs and aggregates are more than an order of magnitude greater than the similar-sized solid spheres. Algal aggregates appear to be highly porous and fractal with a fluid collection efficiency of $10-40 \%$, which would significantly enhance the flocculation between particles and the mass transfer into the aggregates.
\end{abstract}

Keywords Aggregate; Flocculation; Hydrodynamics; Particle; Particle image velocimetry (PIV); Permeability.

\section{INTRODUCTION}

Flocculation, which aggregates small particles into larger and fast-settling flocs, is essential for the transport of particulate matter in natural waters and solid-liquid separation in water and wastewater treatment. The hydrodynamic properties of particles affect the interaction and hence the flocculation kinetics between suspended particles. Characterisation and modelling of the hydrodynamics of individual particles and aggregates are rather difficult because of the small scale of the fluid field, a wide range of particle sizes and the dynamic situation of moving particles in water. However, due to its practical importance, this subject has been intensively studied over the years (Alder, 1981; Han and Lawler, 1992; Veerapaneni and Wiesner, 1996; Li and Logan, 1997, 2001; Tsou et al., 2002).

The classical description of the interaction between approaching particles is the rectilinear collision model, which is known to overpredict the collision frequency function of particles (Han and Lawler, 1991; Li and Logan, 1997). The curvilinear collision models are believed to be more accurate than the rectilinear model in predicting the coagulation and flocculation rates between interactive particles (Jackson and Burd, 1998; Zhang and Li, 2003). However, the curvilinear models do not include a mechanism for the intra-aggregate flow. Aggregates formed by particle flocculation are higher porous and fractal (Logan and Wilkinson, 1990; Jackson and Burd, 1998). Large porous-fractal aggregates will permit greater interior flow through the aggregates, bringing about more particle collisions than predicted by the curvilinear model.

Theoretical characterisation of the aggregate permeability has been carried out by many researchers 
(Alder, 1981, Dullien, 1992; Veerapaneni and Wiesner, 1996; Li and Logan, 1997). In addition, conventional column experiments have been conducted to determine indirectly the permeability of settling aggregates (Lee et al., 1996; Li and Logan, 1997; Li et al., 2003). However, the settling tests are unable to provide more direct streamline information about the hydrodynamic features of the aggregates. Tsou et al. (2002) made a great attempt to visualize the flow field around falling aggregates using a bubble-tracking technique. Nonetheless, air bubbles are hardly considered to be the best flow tracers as they are subject to significant slip from the flow when the bubbles rise to the water surface. In the present experimental study, an advanced flow visualisation technique of particle image velocimetry (PIV) was utilised to study the hydrodynamic properties of settling particles and aggregates in water. The PIV system was able to capture detailed fluid information surrounding the falling objects, which gave the streamlines around the particles and internal permeation through the aggregates.

\section{METHODS AND MATERIALS}

\section{Particle Image Velocimetry (PIV)}

Particle Image Velocimetry (PIV) is a whole-flow-field optical technique that provides instantaneous velocity vector measurements in a cross-section of a flow (Raffel et al., 1998). The PIV system is able to capture the velocity field within a millisecond and to study the flow sensitive to small perturbations. The use of laser illumination, modern CCD cameras and dedicated computing hardware enables the PIV to produce high-quality, real-time flow velocity illustrations.

In the present work, the settling experiments were conducted in a settling column which is a rectangular glass column with a dimension $(\mathrm{L} \times \mathrm{W} \times \mathrm{H})$ of $36 \times 33 \times 350 \mathrm{~mm}$. Individual particles or aggregates were introduced from the top of the column. The present PIV was based on the particle tracking technique which is more effective than the correlation technique for flows of low-density seeding particles. A laser beam was generated and expanded to a laser sheet by a combination of cylindrical and spherical lens (Figure 1). During a settling test, the laser sheet illuminated a thin vertical section (thickness $<0.5 \mathrm{~mm}$ ) through the falling object in the settling column. Polyamid seeding particles (Dantec Dynamic) with diameters around $20 \mu \mathrm{m}$ were suspended in the settling column both as flow tracers and fine particles for flocculation with the falling objects. The motions of the seeding tracers induced by a large settling object were captured by a high-speed CCD camera with a resolution of $1280 \times 1024$ pixels (1200.hs, PCO.imaging). The video images were continuously recorded and transmitted to a workstation for storage and data processing.

\section{Particles and aggregates}

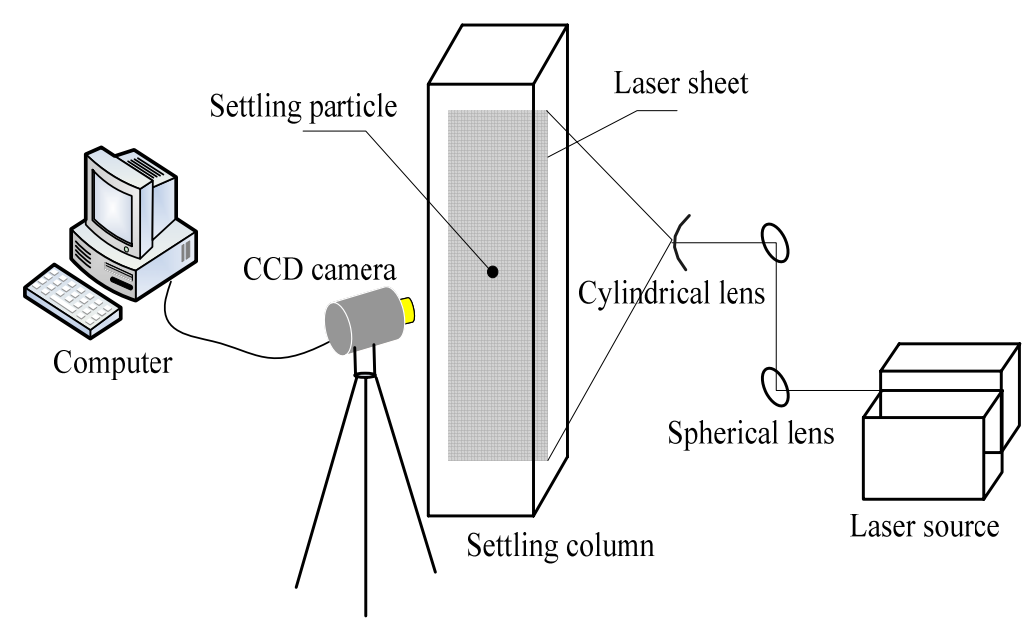

Figure 1. Schematic of the PIV-settling experimental setup.

Three types of particles, including solid spheres, the flocs of small latex microspheres and the aggregates of marine diatom cells, were characterised for their hydrodynamic behaviours during 
settling. Large solid spheres were 400 and $1000 \mu \mathrm{m}$ in size with a density of $\rho=1.05 \mathrm{~g} / \mathrm{cm}^{3}$ (Duke Scientific). The particle flocs sizing from 800 to $2000 \mu \mathrm{m}$ were generated from standard latex microspheres $\left(2-3 \mu \mathrm{m}, \rho=1.05 \mathrm{~g} / \mathrm{cm}^{3}\right.$, Polysciences) by flocculation on a jar-test device (PB-700, Phipps \& Bird) following the procedure described elsewhere (Zhang and Li, 2003). Marine diatoms of Thalassiosra, Chaetoceros gracilis were cultured in the $\mathrm{f} / 2$ growth media with Si (Guillards) in seawater (salinity $=30 \mathrm{ppt}$ ) in an incubator at $22^{\circ} \mathrm{C}$. The diatoms grew into long chains of cells with a cell size of around $10 \mu \mathrm{m}$. The diatom aggregates ranging from 1000 to $2500 \mu \mathrm{m}$ were formed also by jar-test flocculation.

\section{PIV-settling experiments}

In a settling experiment, a particle or aggregate was gently placed at the top of the settling column. The object could reach its terminal falling velocity, $U_{t}$, within $5 \mathrm{~cm}$ from the top of the column. While the object was approaching its terminal velocity of free settling, the column might be adjusted slightly to ensure the illumination of the laser sheet through the centre of the falling object. As described previously, when the large object fell through the view of the CCD camera, the movements of the seeding tracers brought about by the falling object were recorded. The positions of the tracers in relation to the falling object could be identified from the PIV images, and the trajectories of appropriate tracers were tracked which gave the streamlines of flow around and through the falling object. The settling experiments were repeated for a number of particles or aggregates of the same type. Subsequently, a group of streamlines surrounding and through a typical type of falling objects could be determined.

The projected image of each falling object was analyzed for its size and shape factor using a computer-based image analysis system (Scion Image). For an aggregate of irregular shaper, its size is defined as the projected area $(A)$ equivalent diameter, e.g. $d=\sqrt{4 A / \pi}$. The internal permeation of the aggregate may be measured by its fluid collection efficiency $(\eta)$, which is the ratio of the flow passing through the aggregate to the flow approaching the aggregate (Veerapaneni and Wiesner, 1996). The fluid collection efficiency can be estimated from the streamlines around and through the aggregate, or $\eta=\left(d_{i} / d\right)^{2}$, where $d_{i}$ is the span of the streamlines flowing into the aggregate relative to the span of the streamlines $(d)$ approaching it. In addition, a curvilinear reduction factor may be used to relate the curvilinear collision frequency function, $\beta_{\text {cur }}$, with the conventional rectilinear collision frequency function, $\beta_{r e c}$, in $E_{c u r}=\beta_{c u r} / \beta_{r e c}$. The $E_{c u r}$ value can be calculated based on the curvilinear collision model of Han and Lawler (1992). Using the PIV-based streamlines, $E_{c u r}$ for a large falling particle or aggregate can be estimated from $E_{c u r}=\left(\frac{d_{i}+d_{s}}{d+d_{s}}\right)^{2}$, where $d_{s}$ is the size of the seeding tracers $(20 \mu \mathrm{m})$ filled in the settling column.

\section{RESULTS AND DISCCUSION}

\section{Particle tracking}

The PIV results indicate that the particle tracking technique is able to capture the trajectories of the tracers relative to a falling particle or aggregate. Based on the movements of the seed tracers induced by a falling object, the streamlines around and through the object can be defined. Consequently, the permeability and hydrodynamic features of the particles and aggregates of different types can be determined from the streamlines. 

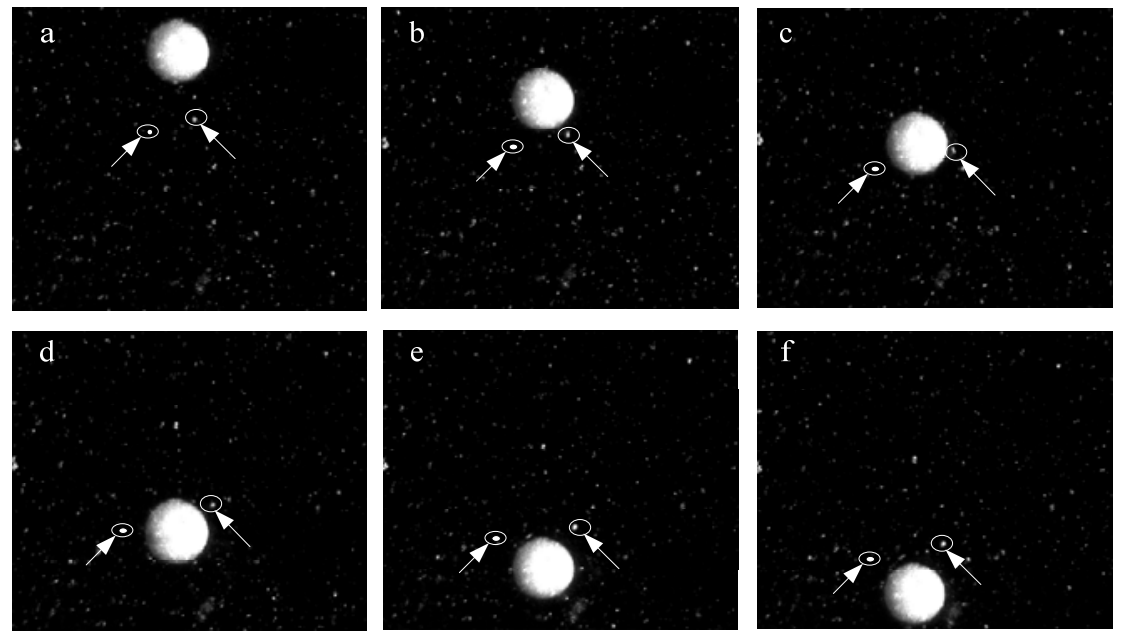

Figure 2. PIV images of a falling solid sphere and the motions of the tracers induced by the falling sphere.

With the illustration of the particle tracers, creeping flow around the falling objects can be well observed, which demonstrate clearly the curvilinear feature of the interaction between approaching particles. The six image frames in Figure 2 show the settling of a large sphere and the motions of the tracers induced by the settling sphere. The sphere $1000 \mu \mathrm{m}$ in diameter had a falling velocity of $10 \mathrm{~mm} / \mathrm{s}$, corresponding to a Reynolds number of 10 . The seeding tracers well away from the path of the falling sphere stayed motionless during the fall of the sphere. However, the two tracers marked in the photos positioning initially below the settling sphere were pushed away from the path of the falling sphere. After the passage of the sphere, the two tracers returned to region in falling path of the large sphere. Following the trajectories of the two tracers, two streamlines can be outlined in relation to the falling sphere. With repeated tests for the same type of spheres, a sufficient number of streamlines can be obtained to define the flow field surrounding the settling spheres.
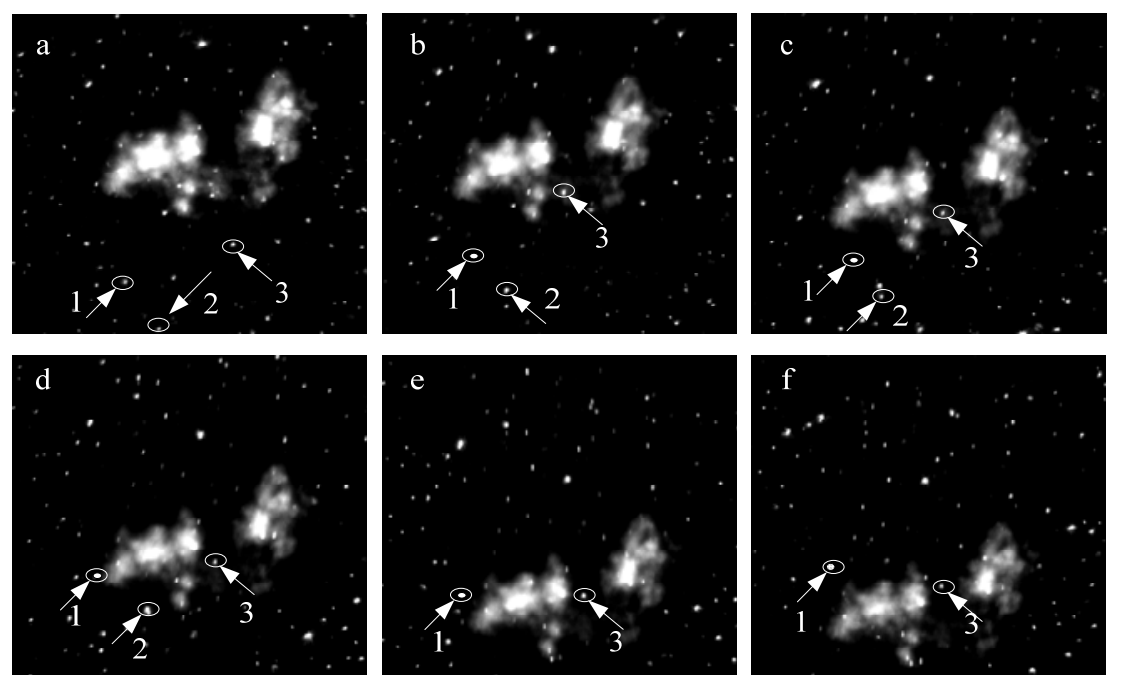

Figure 3. PIV images of a falling diatom aggregate and the motions of the tracers induced by the falling aggregate. 
The particle video and images reveal more complicated fluid situations for particle flocs and aggregates. From the six frames in Figure 3 for a falling diatom aggregate, the motions of three seeding tracers induced by the falling aggregate can be identified. The aggregate having a diameter of $1940 \mu \mathrm{m}$ settled at a velocity of $4.54 \mathrm{~mm} / \mathrm{s}$, corresponding to a Reynolds number of 8.48 . While the aggregate was falling through, tracer 1 just slipped around the surface of the aggregate, and tracers 2 and 3 appeared to be swept by the falling aggregate. As tracer 2 was apparently trapped by the aggregate, tracer 3 came out from the top of the aggregate. Beside the curvilinear feature of the streamlines around falling aggregates, the particle images provide direct evidence of the internal permeation through large and porous aggregates. The findings suggest that the permeable feature of porous and fractal aggregates should be taken into account in description of the particle flocculation kinetics and the mass transport dynamics.

\section{Streamline determination}

Streamlines for solid spheres. Figure 4 include the streamlines around three falling solid spheres determined from particle tracking. The streamlines are creeping and curvilinear lines surrounding the spheres. From the streamlines, the curvilinear reduction factors of the solids spheres during settling can be estimated (Table 1). The reduction factors in relation to the conventional rectilinear model decreases as the Reynolds number increases. This implies that the tracers had a less opportunity to collide with the spheres as the falling velocities of the solid spheres increase. This tendency is in agreement with the predication of the curvilinear model given by Han and Lawler (1992). However, it also can be noted that the reduction factors estimated from the PIV results are one or two orders of magnitude higher than those predicated by the curvilinear model. It is apparent that the curvilinear model underestimates the collision frequency between approaching particles.
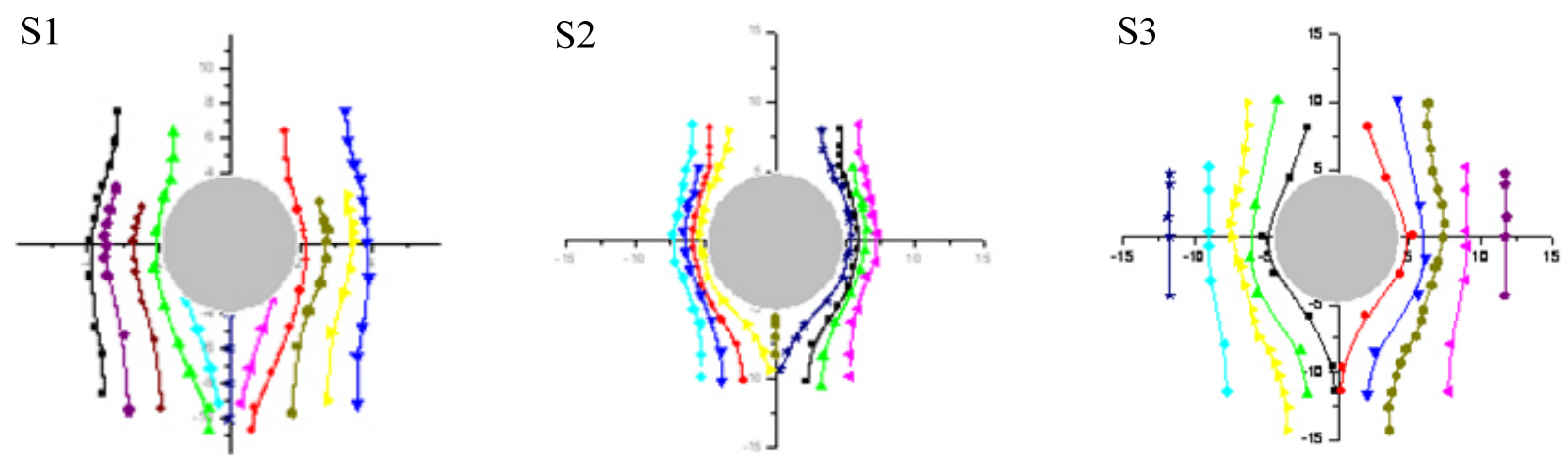

Figure 4. Streamlines around falling solid spheres: (S1) $400 \mu \mathrm{m}$, (S2) $1000 \mu \mathrm{m}$ and (S3) $1000 \mu \mathrm{m}$.

Table 1. PIV results for the falling solid spheres.

\begin{tabular}{cccccccc}
\hline ID & $\begin{array}{c}\text { Salinity } \\
(\mathrm{g} / L)\end{array}$ & $\begin{array}{c}\text { Fluid } \\
\text { density } \\
\left(\mathrm{g} / \mathrm{cm}^{3}\right)\end{array}$ & $\begin{array}{c}\text { Diameter } \\
(\mu \mathrm{m})\end{array}$ & $\begin{array}{c}U_{t} \\
(\mathrm{~mm} / \mathrm{s})\end{array}$ & $R e$ & $\begin{array}{c}\text { Curvilinear } \\
\text { reduction factor } \\
(\text { Expt. })\end{array}$ & $\begin{array}{c}\text { Curvilinear } \\
\text { reduction factor } \\
(\text { model })\end{array}$ \\
\hline $\mathrm{S} 1$ & 30 & 1.024 & 400 & 2.0 & 0.8 & 0.090 & 0.00026 \\
$\mathrm{~S} 2$ & 40 & 1.032 & 1000 & 10.0 & 10 & 0.013 & 0.00018 \\
$\mathrm{~S} 3$ & 20 & 1.016 & 1000 & 18.0 & 18 & $\approx 0.001$ & 0.00012 \\
$\mathrm{~S} 4$ & 30 & 1.024 & 1000 & 14 & 14 & 0.009 & 0.00015 \\
\hline
\end{tabular}


Streamlines for the flocs of latex microspheres. Figure 5 gives the streamlines surrounding the settling flocs of latex microspheres. There are three types of the trajectories of the tracers that may be identified from the PIV images. The first type shows the streamlines around the falling flocs, which is similar to the case of previous solid spheres. The second type signifies the tracers that penetrated into the flocs and were captured by the flocs. The third type shows the tracers that entered and passed through the flocs without attachment. All of the streamlines demonstrate the curvilinear movements of the seed particles relative to the settling of the large flocs. More importantly, the PIV result provides a direct proof of the internal permeation through large and fractal particle flocs. The fluid collection efficiency ranges from 0.06 to 0.18 for the flocs examined (Table 2). In other words, more than $6 \%$ of the fluid approaching the flocs flowed into the interior of the flocs. The curvilinear reduction factors varied from 0.07 to 0.18 , which are more than one order of magnitude greater than those of the solid spheres. Thus, the internal flow through permeable flocs can significantly enhance the collisions of the flocs with other suspended particles.
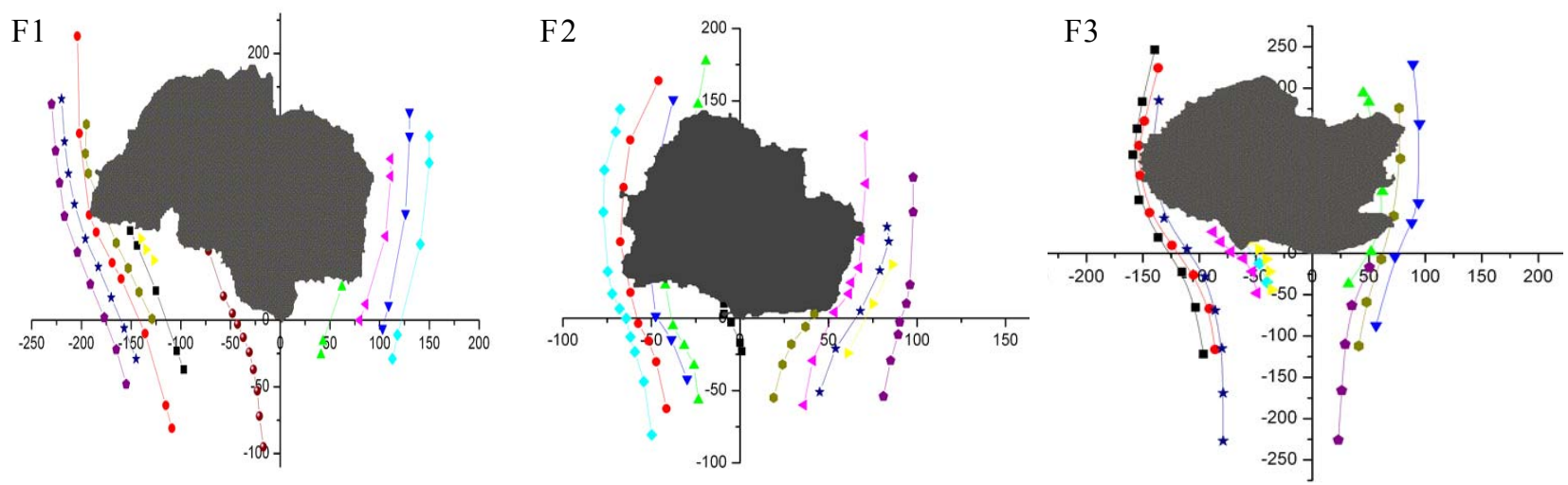

Figure 5. Streamlines around falling particle flocs: (F1) $2172 \mu \mathrm{m}$, (F2) $1406 \mu \mathrm{m}$ and (F3) $1688 \mu \mathrm{m}$.

Table 2. PIV results for the falling microsphere flocs.

\begin{tabular}{cccccccc}
\hline ID & $\begin{array}{c}\text { Diameter } \\
(\mu \mathrm{m})\end{array}$ & $\begin{array}{c}\text { Shape } \\
\text { factor }\end{array}$ & $\begin{array}{c}U_{t} \\
(\mathrm{~mm} / \mathrm{s})\end{array}$ & $R e$ & $\begin{array}{c}\text { Curvilinear } \\
\text { reduction factor } \\
(\text { expt. })\end{array}$ & $\begin{array}{c}\text { Curvilinear } \\
\text { reduction } \\
\text { factor (model) }\end{array}$ & $\begin{array}{c}\text { Fluid } \\
\text { collection } \\
\text { efficiency }\end{array}$ \\
\hline F1 & 2172 & 0.20 & 3.5 & 7.6 & 0.18 & 0.00100 & 0.18 \\
F2 & 1406 & 0.30 & 1.5 & 2.1 & 0.11 & 0.00036 & 0.10 \\
F3 & 1688 & 0.24 & 4.6 & 4.6 & 0.13 & 0.00038 & 0.12 \\
F4 & 1194 & 0.17 & 3.6 & 4.2 & 0.07 & 0.00040 & 0.06 \\
F5 & 1438 & 0.44 & 3.0 & 4.3 & 0.10 & 0.00037 & 0.09 \\
\hline
\end{tabular}

Streamlines for diatom aggregates. A similar hydrodynamic phenomenon was observed for the aggregates of marine diatom cells. Figure 6 shows the PIV streamlines around and through the falling aggregates. Compared to the previous particle flocs, the diatom aggregates appeared to be much more porous and permeable. The fluid collection efficiencies of the individual aggregates ranged from 0.08 to 0.42 (Table 3), which in general are greater than those of the microsphere flocs. The significant internal flow is beneficial to the mass transport into the aggregates and their flocculation with other particles. The curvilinear reduction factors of the aggregates estimated from the PIV results are generally three orders of magnitude greater than predicted by the curvilinear model (Han and Lawler, 1992). Therefore, the importance of the internal permeation to mass transfer and particle interaction cannot be neglected for the highly porous algal aggregates. 

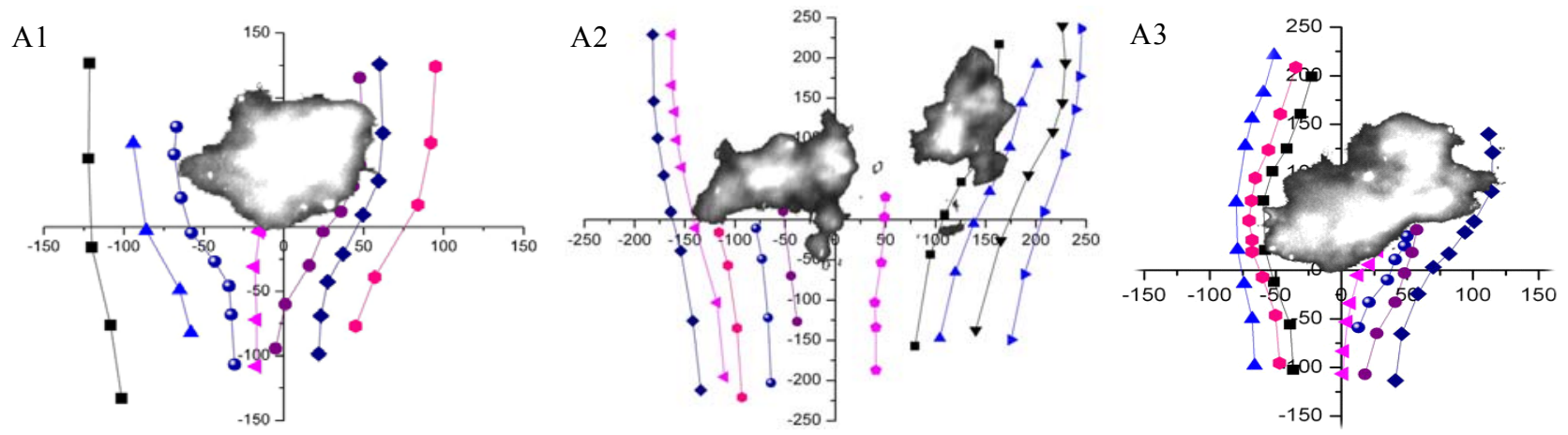

Figure 6. Streamlines around diatom aggregates: (A1) $844 \mu \mathrm{m}$, (A2) $1940 \mu \mathrm{m}$ and (A3) $1257 \mu \mathrm{m}$.

Table 3. PIV results for the falling diatom aggregates.

\begin{tabular}{cccccccc}
\hline ID & $\begin{array}{c}\text { Diameter } \\
(\mu \mathrm{m})\end{array}$ & $\begin{array}{c}\text { Shape } \\
\text { factor }\end{array}$ & $\begin{array}{c}U_{t} \\
(\mathrm{~mm} / \mathrm{s})\end{array}$ & $R e$ & $\begin{array}{c}\text { Curvilinear } \\
\text { reduction factor } \\
(\text { expt.) }\end{array}$ & $\begin{array}{c}\text { Curvilinear } \\
\text { reduction factor } \\
\text { (model) }\end{array}$ & $\begin{array}{c}\text { Fluid } \\
\text { collection } \\
\text { efficiency }\end{array}$ \\
\hline A1 & 844 & 0.54 & 2.15 & 1.75 & 0.13 & 0.00049 & 0.12 \\
A2 & 1940 & 0.32 & 4.54 & 8.48 & 0.42 & 0.00034 & 0.42 \\
A3 & 1257 & 0.27 & 2.69 & 3.25 & 0.21 & 0.00039 & 0.20 \\
A4 & 986 & 0.10 & 3.25 & 3.20 & 0.36 & 0.00045 & 0.35 \\
A5 & 2021 & 0.04 & 5.84 & 11.79 & 0.08 & 0.00031 & 0.08 \\
\hline
\end{tabular}

\section{Aggregate permeability and its implication}

Streamlines for falling solid spheres, particle flocs and diatom aggregates all demonstrate the curvilinear nature of the interactions between approaching particles. The PIV results provide direct evidence that the rectilinear model overpredicts the collision rates between suspended particles. However, the curvilinear reduction factors calculated based on the curvilinear collision model of Han and Lawler (1992) are about two orders of magnitude lower than those estimated from the experimental results. This suggests that the available curvilinear model may underestimate the collision potential between particles to a great extent. Aggregates formed by particle flocculation are highly porous and fractal. The flocs of microspheres could have a fractal dimension varying from 1.8 to 2.2 (Logan and Wilkinson, 1990, Li and Logan, 1997). Marine aggregates can be more fractal with a lower value of fractal dimension ranging from 1.3 to 2.0 (Logan and Wilkinson, 1990). The lower is the fractal dimension, the more porous and permeable are the aggregates. In the present PIV investigation, both the particle flocs and diatom aggregates have curvilinear reduction factors about two orders of magnitude greater than those of similarly-sized solid spheres. The curvilinear reduction factors of the diatom aggregates are even higher than those of the particle flocs. It is evidenced that the porous-fractal structure of the aggregates allows flow through the aggregate interior. The significant internal permeation can greatly enhance the flocculation of aggregates with other particles as well as the material transfer into the aggregates. The PIV results also suggest that the fluid collection efficiency is closely related to the interior structure of the aggregates, which requests additional characterisation. 


\section{CONCLUSIONS}

The fluid field around falling particles, including solid spheres, particle flocs, and diatom aggregates, can be directly observed and recorded using the particle-tracking based PIV technique. The experimental study validates the curvilinear feature of the interaction between approaching particles. The trajectories of the seed tracers relative to a large falling particle or aggregate outline the streamlines surrounding the falling object. The PIV streamlines provide a direct experimental proof of the internal flow through particle flocs and algal aggregates. The available curvilinear model underestimates the collision frequency function by one order of magnitude or two for solid spheres. The collision potentials of the aggregates are more than an order of magnitude greater than the similar-sized spheres. Algal aggregates appear to be highly porous and fractal, which allows streamlines to penetrate into the aggregate interior with a fluid collection efficiency of $10-40 \%$. The permeable feature of particle aggregates can significantly enhance the collisions and flocculation between particles and mass transfer into the aggregates.

\section{ACKNOWLEDGMENTS}

This research was supported by grants HKU7120/03E from the Research Grants Council (RGC) and AoE/P-04/2004 from the University Grants Committee (UGC) of the Hong Kong SAR Government, China. The technical assistances of Mr. Keith C. H. Wong and Mr. C.H. Tong are greatly appreciated.

\section{REFERENCES}

Alder P.M. (1981). Stremalines in and around porous particles. J. Colloid Interface Sci., 81, 531-535.

Dullien F.A.L. (1992). Porous Media Fluid Transport and Pore Structure. $2^{\text {nd }}$ edn, Academic Press:San Diego.

Jackson G.A. and Burd A.B. (1998). Aggregation in the marine environment. Environ. Sci. Technol., $32,2805-2814$.

Han M. and Lawler D.F. (1992). The (relative) insignificance of $\mathrm{G}$ in flocculation. $J . A W W A$, 84(10), 79-91.

Lee D. J., Chen G. W., Liao Y. C. and Hsieh C. C. (1996). On the free-settling test for estimating activated sludge floc density. Water Res., 30, 541-550.

Li X.Y. and Logan B.E. (1997). Collision frequencies between fractal aggregates and small particles by differential sedimentation. Environ. Sci. Technol., 31, 1229-1236.

Li X.Y. and Logan B.E. (2001). Permeability of fractal aggregates. Water Res., 35, 3373-3380.

Li X.Y., Yuan Y. and Wang H.W. (2003). Hydrodynamics of biological aggregates of different sludge ages: an insight into the mass transport mechanism of bioaggregates. Environ. Sci. Technol., 37, 292-299.

Logan B.E. and Wilkinson D.B. (1990). Fractal geometry of marine snow and other biological aggregates. Limnol. Oceanogr., 35, 130-136.

Raffel M., Willert C.E. and Kompenhans J. (1998). Particle Image Velocimetry: A Practical Guide. Springer:Berlin.

Tsou G.W., Wu R.W., Yen P.S., Lee D.J. and Peng X.F. (2002). Advective flow and floc permeability. J. Colloid Interface Sci., 250, 400-408.

Veerapaneni S. and Wiesner M.R. (1996). Hydrodynamics of fractal aggregates with radially varying permeability. J. Colloid Interface Sci., 177, 45-57.

Zhang J.J. and Li X.Y. (2003). Modeling particle-size distribution dynamics in a flocculation system. AIChE J., 49, 1870-1882. 